\title{
El desarrollo de competencias investigativas en la formación de estudiantes de la UNED de Costa Rica mediante la metodología Lean Startup
}

The development of research competences through the Lean Startup methodology in students training at the State Distance University in Costa Rica

\author{
Carolina Ávalos Dávila*1 \\ cavalos@uned.ac.cr \\ María luisa Sevillano García** \\ m/sevillano@edu.uned.es. \\ * Universidad Estatal a Distancia, Costa Rica \\ **Universidad Nacional de Educación a Distancia, España
}

\section{Resumen:}

Se realiza un análisis de los planteamientos del método Lean Startup para ser usados en la creación de una metodología de aprendizaje orientada al desarrollo de competencias investigativas en la formación de estudiantes de la UNED de Costa Rica. Aspecto a reforzar como recomendación de instancias superiores universitarias, desde la formación del estudiantado costarricense.

Para ello, se analizan y contrastan investigaciones desarrolladas en el tema de Lean Startup por expertos estadounidenses y europeos sobre experiencias logradas y su aplicación en la docencia.

Resaltamos, que las competencias em-

\begin{abstract}
:
This study performs an analysis of the Lean Startup method to check whether some of its maxims can be used in the creation of a learning methodology oriented to the development of research competences in the training of students at the Open University in Costa Rica. The reinforcement of this aspect has been highlighted as a priority by university authorities. To do this, we analyse and contrast research on the Lean Startup methodology by American and European experts which covers experiences and their application to teaching. The entrepreneurial skills achieved by working with the Lean Startup methodology help incorporate research actions that
\end{abstract}

1 Dirección para correspondencia (correspondence address):

Carolina Ávalos Dávila. Facultad de Educación UNED. Edificio C, 2do. Piso. De la Rotonda La Betania 500m al este, Carretera a Sabanilla Mercedes de Montes de Oca. San José, 474-2050. Campus de la Universidad Estatal a Distancia (Costa Rica). 
El desarrollo de competencias investigativas en la formación de estudiantes de la UNED de Costa Rica mediante la metodología Lean Startup

Carolina Ávalos Dávila y María luisa Sevillano García

prendedoras logradas al trabajar con la metodología Lean Startup, incorporan el desarrollo de acciones investigativas que deberían estar presentes en la formación de investigación del estudiantado de la UNED y simultáneamente, se relacionan con las competencias básicas que todo estudiante debe tener en investigación desde planteamientos de Cuevas y Rocha (2011) y Davison \& Palermo (2015).

Consideramos que se forman estudiantes con actitudes emprendedoras al usar los planteamientos del método Lean Startup desde sus tres pasos: crear- medir- aprender, siendo posible además, el desarrollo intrínseco de competencias investigativas como resultado de una formación emprendedora. Esto nos informa, de la efectividad de adecuar el método Lean Startup al campo de la docencia, con el interés de formar futuros profesionales desde una metodología de aprendizaje enfocada al desarrollo de actitudes emprendedoras, siendo coadyuvantes en una formación de competencias investigativas desde el inicio de su carrera universitaria en la UNED.

\section{Palabras clave:}

Lean Startup; emprendimiento; competencias en investigación; metodología de aprendizaje; Educación. should be present in the research training of Open University students. Simultaneously, the acquisition of these research actions relate to the basic competences that every student should have in research (Cuevas and Rocha, 2011; Davison \& Palermo, 2015). We consider that the three steps of the Lean Startup methodologycreating-measuring-learning-do not only help train enterprising future teachers but also develop research competencies. This informs us of the effectiveness of adapting the Lean Startup method to the field of teaching, with a view to training future professionals according to a learning methodology focused on the development of entrepreneurial attitudes which, simultaneously, help students acquire research competences from the beginning of their university degrees at the Open University.

\section{Key words:}

Lean Startup; entrepreneurship; research competences; learning methodology; Education.

\section{Résumé:}

Une analyse est faite des approches de la méthode Lean Startup à utiliser dans la création d'une méthodologie d'apprentissage orientée vers le développement des compétences de recherche dans la formation des étudiants de I'UNED du Costa Rica. Aspect à renforcer comme recommandation des instances universitaires supérieures, à partir de la formation des étudiants costariciens.

Pour ce faire, nous analysons et contredisons les recherches développées dans Lean Startup par des experts américains et européens sur les expériences et leur application dans l'enseignement.

Dans un même temps, les compétences entrepreneuriales acquises en travaillant avec la méthodologie Lean Startup intègrent le développement d'actions de recherche qui devraient être présentes dans la formation de recherche des étudiants de I'UNED et, simultanément, les compétences de base que chaque étudiant devrait avoir dans la recherche Cuevas et Rocha (2011) et Davison et Palerme (2015).

Nous considérons que les étudiants sont formés avec des attitudes entrepreneuriales lorsqu'ils utilisent les approches de la méthode Lean Startup à partir de ces trois étapes : construire-mesurer-apprendre, étant également possible, le développement intrinsèque des compétences d'enquête résultant d'une formation entrepreneuriale.

Cela nous informe de l'efficacité de l'adaptation de la méthode Lean Startup au domaine de l'enseignement, avec l'intérêt de former de futurs professionnels à partir d'une méthodologie d'apprentissage centrée sur le développement des attitudes entrepreneu- 
riales, adjuvantes d'une formation aux compétences d'investigation dès le début de la carrière universitaire à l'UNED.

\section{Mots clés:}

Lean Startup; entrepreneuriat; compétences en recherche; méthodologie d'apprentissage; éducation.

Fecha de recepción: 15-1-2018

Fecha de aceptación: 28-1-2018

\section{Introducción}

Todos los días la sociedad impone cambios e innovaciones las cuales, sin que el ser humano las determine, van siendo parte del diario cotidiano e impulsan a una mejora de las diferentes capacidades desarrolladas por las personas.

Ante el panorama que se evidencia, las universidades como centros de formación para los futuros profesionales, tienen el reto de formar estudiantes pensando en que podrán ser actores sociales de cambio para el futuro, sin embargo, con el pasar de los años, según, Peñalver (2017), se ha notado la repetición de metodologías de enseñanza usadas en siglos pasados, formando con ello, un perfil de estudiante que va muy desfasado en relación con las demandas de la actual sociedad del conocimiento.

Considerando que una de las recientes tendencias descubiertas e implementadas en el área de Administración es el logro de competencias emprendedoras, la cual fomenta en los empresarios el incremento de sus capacidades de negociación, pensamiento creativo, reflexivo e indagador y va acorde con las actuales demandas de la sociedad en la búsqueda de un profesional proactivo, que resuelve problemas, líder y comprometido con su quehacer (Ries, 2011), es por lo que se analizará la filosofía que plantea el método Lean Startup con su metodología enfocada en: crear-medir- aprender.

Si bien es cierto, se han evidenciado desde la opinión de diferentes estudiosos en el tema, tal es el caso de: Colapinto (2013), Blank (2013), Gutpe (2015), Sousa y Cavalcanti (2016), entre otros, buenas experiencias empresariales y de formación, producto de la implementación del método Lean Startup y es a partir de ello, que interesa reflexionar desde la presente investigación, los beneficios que se podrían obtener al im- 
plementar dicho método en la formación profesional de los estudiantes de la Escuela de Ciencias de Educación de la UNED Costa Rica, como metodología de aprendizaje que facilite la generación de actitudes emprendedoras y consecuentemente, el desarrollo de competencias en investigación tan requeridas actualmente, en la formación de profesionales docentes.

Pretendemos analizar aquí, los elementos educativos que se requieren para la puesta en marcha del método, su transformación en metodología de enseñanza y las implicaciones de considerar dicho planteamiento como plan remedial en atención a las carencias existentes en la formación investigativa del estudiantado de la UNED.

\section{Método}

La metodología considerada en la investigación es la interpretativa de tipo documental, que permite el estudio del conocimiento acumulado escrito en un tema determinado. (Gómez, Galeano y Jaramillo, 2015).

Para su puesta en marcha se analizaron alrededor de 90 documentos de diverso tipo: artículos científicos, artículos de opinión, trabajos de investigación de grado y posgrado, manuales, folletos, libros, entrevistas, blogs personales y videos, en idioma español, inglés y portugués, relacionados al método Lean Startup, emprendimiento y experiencias sobre el uso del método como metodología empleada en diferentes áreas de formación.

El tratamiento de la información consistió en el diseño de fichas de lectura de la documentación consultada, el contraste de la información obtenida que luego fue esquematizada para hacer una selección considerando el tema central de la investigación y sus alcances, aspectos reflejados en la fundamentación teórica.

\section{Fundamentación teórica}

\section{La Competencia Investigativa en Educación Superior}

La sociedad del conocimiento desde sus innovaciones permanentes, sugiere la reflexión sobre los perfiles de salida y formación profesional 
que reciben los estudiantes en sus diferentes áreas de formación. En la actualidad, para C. Tünnermann y Souza (2003), los cambios sociales, culturales, económicos y políticos producen una serie de transformaciones tanto en espacios cotidianos de las personas como de las diferentes organizaciones e instituciones, entre ellas, las de educación superior.

Sin embargo, desde las aulas, en aspectos de mediación, evaluación y aprendizaje significativo, la realidad dista mucho de lo que se sugiere en la normativa educativa institucional y las acciones evidenciadas se direccionan a generar, en última instancia, aprendizajes desechables para el estudiante. Desde el campo de la educación, la investigación como quehacer, ocupa los últimos lugares en cuanto a la cantidad de producciones que se Ilevan a cabo a nivel nacional. (Consejo Nacional de Rectores, 2016).

Los enunciados anteriores, ejercen una preocupación para las autoridades universitarias, quienes, en vista de su responsabilidad social, están obligadas a ejercer la función investigativa desde áreas como la docencia y extensión, convirtiéndose en una entidad formadora que incentive el cambio, la mejora y proyección sociocultural.

Es así como la Universidad Estatal a Distancia de Costa Rica, a partir de su normativa institucional y políticas educativas, en palabras de Calderón (2013), tiene el reto de desarrollar una formación investigativa en el estudiantado desde los diferentes planes y programas de estudio.

Por su parte, formar en investigación debe entenderse como un proceso permanente de prácticas que involucra a actores diversos, concretándose en un quehacer académico que promueva el acceso a conocimientos, desarrollo de habilidades, destrezas y actitudes, así como la interiorización de valores, los cuales se materializan en la realización de una actividad investigativa según Moreno (2005) y Maldonado, et al. (2007), donde dicha práctica se apoya en diversos procedimientos, partiendo de un objetivo fundamental que la orienta.

Desde la reflexión y considerando el real interés que tiene la investigación como práctica académica, autores como: Moreno (2005), Guerrero (2007), Cuevas y Rocha (2011), Tobón (2013), y Davison \& Palermo (2015), coinciden en que las siguientes competencias básicas en investigación (ver tabla 1), son las que deben estar presentes en la formación de los estudiantes universitarios, desde las diferentes áreas de formación: 
Tabla 1. Competencias básicas en investigación.

Competencias El estudiante tiene la capacidad de:

- Conocer las líneas de investigación del programa y desarrollar investigaciones a partir de ellas.

- Concretar conocimiento epistemológico, ontológico y metodológico en investigación educativa.

- Informarse sobre cómo ampliar o mejorar su investigación

Organizativas con expertos, inscribirse en proyectos de carácter institucional financiados.

- Desarrollar conciencia de una ética investigativa desde el inicio del planteamiento de la investigación.

- Participar activamente en procesos de extensión e investigación institucionales.

Comunicacio- - Planificar y llevar a cabo proyectos de investigación para nales resolver problemas relevantes del contexto.

- Problematizar y plantear la interrogación como elemento esencial en la construcción de conocimiento.

- Desarrollar pensamiento crítico y autorregulación del pensamiento.

- Reunir información haciendo acopio de datos desde un proceso intencional con dirección y sentido.

- Gestionar el conocimiento, manejo de la información y recursos tecnológicos.

- Asistir a actividades académicas para reforzar su actitud investigadora (Jornadas, coloquios, encuentros.)

- Profundizar el conocimiento en investigación logrado desde la participación en eventos de investigación como congresos.

- Participar activamente en procesos de extensión e investigación desde su comunidad, como gente social de cambio.

- Socializar resultados de investigaciones realizadas solo o en coautoría.

Colaborativas - Socializar las ideas de la investigación, gestionando actitudes de liderazgo y apertura para el trabajo en equipo desde su comunidad de aprendizaje.

- Participar en presentaciones públicas de investigación como oyente o expositor.

- Participar activamente en eventos de investigación con otros expertos para mejorar su quehacer investigativo (congresos, foros, seminarios, conferencias).

- Participar como coautor en proyectos de investigación con otros colaboradores. (Semilleros de Investigación.) 
Las competencias investigativas básicas mencionadas, resultan ser un elemento clave en la formación de los estudiantes y de gran importancia para el desarrollo e incorporación en el campo profesional, personal y social. Se despliegan desde la praxis existente en la metodología empleada, contenidos temáticos, estrategias didácticas y heurísticas que sugieren, todo un proceso dialógico de aprendizaje generado entre los actores educativos.

Pero, además, tener en cuenta el desarrollo de una formación de competencias en investigación, incurre en un alto beneficio para los futuros profesionales; pues para Tobón (2013), resulta sustancial destacar aspectos como:

- Aumento en la pertinencia de programas educativos: donde se orienta el aprendizaje acorde con los retos y problemas del contexto social y disciplinar- investigativo, ofreciendo un sentido más realista al aprendizaje del estudiante.

- La gestión de la calidad: es posible gestionar la calidad desde aspectos como la calidad de desempeño y calidad de formación, de forma que se contribuya aportando indicadores claros para el logro de la calidad, una precisión en los productos a lograr, detectar fallos y evaluar para la mejora en su continua pertinencia.

- Política educativa internacional: la formación en competencias se está convirtiendo en una política educativa de alto alcance, esto permite la generación de contribuciones conceptuales y metodológicas por parte de investigadores de varios países y desarrollo de proyectos internacionales educativos con el apoyo de instituciones educativas.

- Movilidad: para la generación de un sistema donde se reconozcan de forma más clara los conocimientos previos y experticia de expertos investigadores, siendo más fácil identificar las diferentes habilidades, destrezas y capacidades de cada actor educativo, para encausar esos esfuerzos en proyectos donde sus aportes investigativos sean de alto provecho.

\section{Búsqueda de nuevos métodos en educación: Lean Startup en tres pasos: (crear- medir- aprender)}

La educación en la actualidad se concibe como una acción intencionada, global y contextualizada regida por normas personales y sociales; 
donde uno de los elementos que la enriquece es la investigación, que de acuerdo con Albert (2009), posibilita comprender la conducta humana desde los significados e intenciones de los sujetos que intervienen en el escenario educativo.

Por tanto, es al sistema educativo, a quien le corresponde la tarea de preparar a los profesionales que la sociedad requiere, pero es a la universidad a la que le atañe, disponer de las profesiones o crear las que sean convenientes de acuerdo a las demandas de la sociedad, y según aportes de Schank (2011), esto último no se ha venido haciendo, en su defecto, se sigue formando sin sentido, enseñando contenidos que no son significativos para el estudiante en su proceso de formación. Así lo afirma Alabart (sf), citado en Borafull (2014): "el sistema educativo actual obliga a todos a pasar por el tubo de la homogeneización, no considerando talentos individuales e inculcando conocimientos de carácter genérico y no significativos". (p.156).

Lo anterior revela, que en la medida en que se aprendan cosas que le gustan al estudiante, ese conocimiento será más perdurable, pues se hace uso de habilidades innatas en lugar de adquirir nuevas habilidades que no se sepan desarrollar adecuadamente.

Al respecto Hecht (2010), enfatiza que, desde la cognición, prevalece más el interés del cómo se aprende que lo que se ha aprendido, pues se valora más la importancia del cómo, en la medida que demuestra la capacidad para seguir aprendiendo y estar al día en el proceso de formación.

Ante el panorama actual, resulta interesante, animarse a incursionar en la búsqueda de metodologías de aprendizaje innovadoras, tal es el caso de la que se sustenta en los planteamientos del método Lean Startup, pues desde lo que se plantea, las empresas en la actualidad están trabajando en forma de células y no pirámides, donde no prevalece la competencia, sino más bien la comunicación y cooperación entre las mismas, siendo un reto constante para quienes asuman el trabajo en estas células, pues tienen que estar formándose constantemente y aprendiendo un poco de todo y de quienes les rodean a diario, con tal de sacar adelante los proyectos en las empresas. (Borafull, 2014).

Para Blank (2013), Lean Startup," es un método que ha revolucionado la forma de ver el proceso de iniciación de una empresa, haciéndolo menos arriesgado, favoreciendo la experimentación sobre la planificación, 
dándole importancia a la retroalimentación del cliente sobre la intuición, iterando y diseñando sobre el desarrollo tradicional" (párr. 5).

Dicho método fue creado por Erick Ries en el año 2011 en Estados Unidos, su propósito principal radica en "generar negocios prósperos que cambien al mundo" (p.11). Se basa en el incremento de las ganancias de las empresas, altas capacidades de negociación de los emprendedores, desarrollo de pensamiento creativo y generación de productos que respondan a las necesidades de la población, en la concreción de profesionales proactivos, solucionadores de problemas, líderes y de compromiso con su quehacer.

El método se sustenta en un ciclo de tres pasos: Crear - Medir Aprender y es ejecutado actualmente en el área de Administración de empresas, donde se busca que sean minimizados los riesgos a partir de una serie de acciones que consisten en: trabajar con el menor uso de recursos, evaluar la calidad de los productos elaborados, aprender de los errores, pivotar la idea de ser necesario y finalmente, hacer un lanzamiento más efectivo de los productos creados. (Ries, 2011)

El proceso de crear - medir y aprender, ilustrado en la figura 1, se concibe como un sistema escalable que parte de una idea inicial, con el planteamiento de una hipótesis, desde la cual se analiza la viabilidad de su puesta en marcha, mediante diferentes acciones que involucran prácticas de indagación, liderazgo, creatividad, empatía y colaboración entre los involucrados, se generan las oportunidades para medir la pertinencia y factibilidad de la idea en desarrollo o en su defecto, pivotear o cambiar de idea, para finalmente, una vez materializada la idea inicial en el lanzamiento de un producto de interés para el mercado, valorar el camino recorrido, ver sus pro y contras, aprender de los fallos, convertirlos en fortalezas y evitar cometer errores anteriores.

Dicho ciclo pretende, que se usen de forma eficiente los recursos materiales y humanos para minimizar el desperdicio, pues como emprendedores, se requiere transformar las buenas ideas en empresas sólidas que generen valor para los involucrados. (Ries, 2011). 


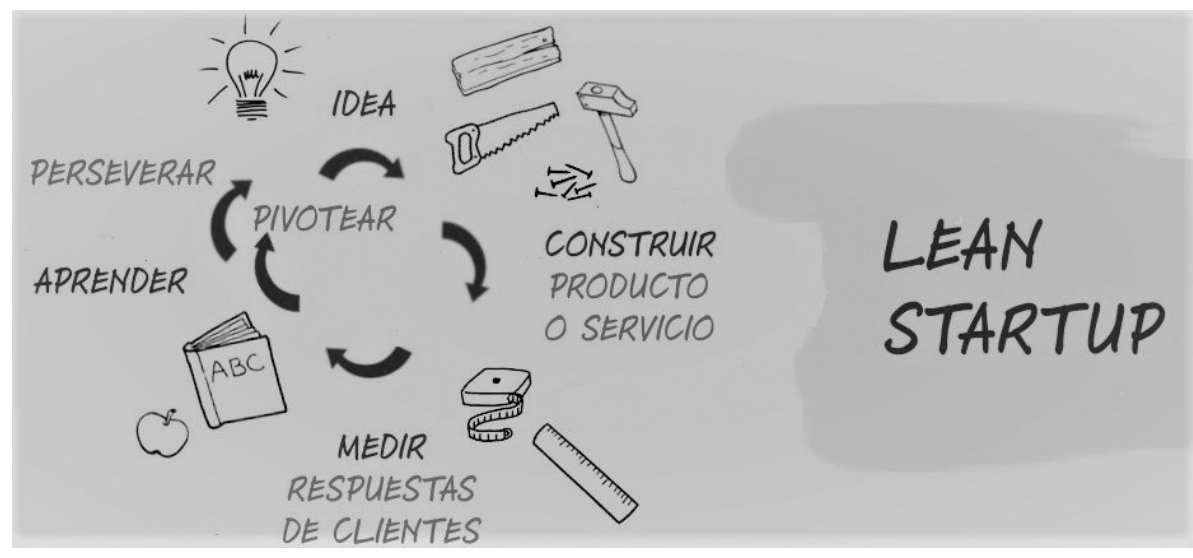

Figura 1. Pasos del método Lean Startup

Fuente: https://www.designthinking.es/comparte/view.php?id=305\&tipo

La clave de los pasos ilustrados está en lograr el desarrollo de un prototipo que permita medir su eficacia en el mercado, aprender del experimento y al menor costo posible.

Producto de lo descrito, surge una metodología basada en el aprendizaje validado, la experimentación científica, la iteración del producto, acortando ciclos de desarrollo, medición del progreso y la obtención de una realimentación a ser considerada en la viabilidad del producto mismo. (Cooper y Vlaskovits, 2014).

Es así como se presenta el interés de analizar las oportunidades que puede brindar este método en el desarrollo de una nueva metodología de aprendizaje que contribuya a la formación de mentes emprendedoras, viendo los errores como procesos de entrenamiento y el conocimiento como una acción metamórfica "que se construye, destruye y reconstruye, de acuerdo con el modelo científico actual, siempre en proceso de modificación, transformándose por la acción del individuo sobre su mundo" (Sevillano, 2011, p.31).

Para el desarrollo de una nueva metodología de aprendizaje apoyada en el método Lean Startup, es necesario contemplar como principales claves de éxito: el pensamiento emprendedor, la predisposición de asumir el fracaso como técnica para aprender, mejorar e innovar de forma permanente y el desarrollo de actitudes colaboradoras.

De igual forma, se ve necesario ir reflexionando en el rediseño de las estrategias didácticas, que vayan enfocadas en el aprender haciendo, aprendizaje por retos y aprendizaje móvil, desarrollando capacidades 
que favorezcan la indagación, creatividad, comunicación efectiva, trabajo colaborativo, cooperativo y la confianza. (Peñalver, 2017).

\section{¿Cómo lograr una formación en investigación a partir de competencias y actitudes emprendedoras?}

A partir de un estudio diagnóstico realizado en el año 2016, a 201 estudiantes de la UNED Costa Rica sobre las competencias investigativas logradas, al finalizar el estudio de las asignaturas de la cátedra de Investigación Educativa de la Escuela Ciencias de la Educación, se encontraron aspectos importantes por reforzar, en el desarrollo de las competencias organizativas, comunicacionales y colaborativas, las cuales se desprenden de la tabla 1 y se retoman con mayor detalle en la figura 2. (Ávalos, 2017).

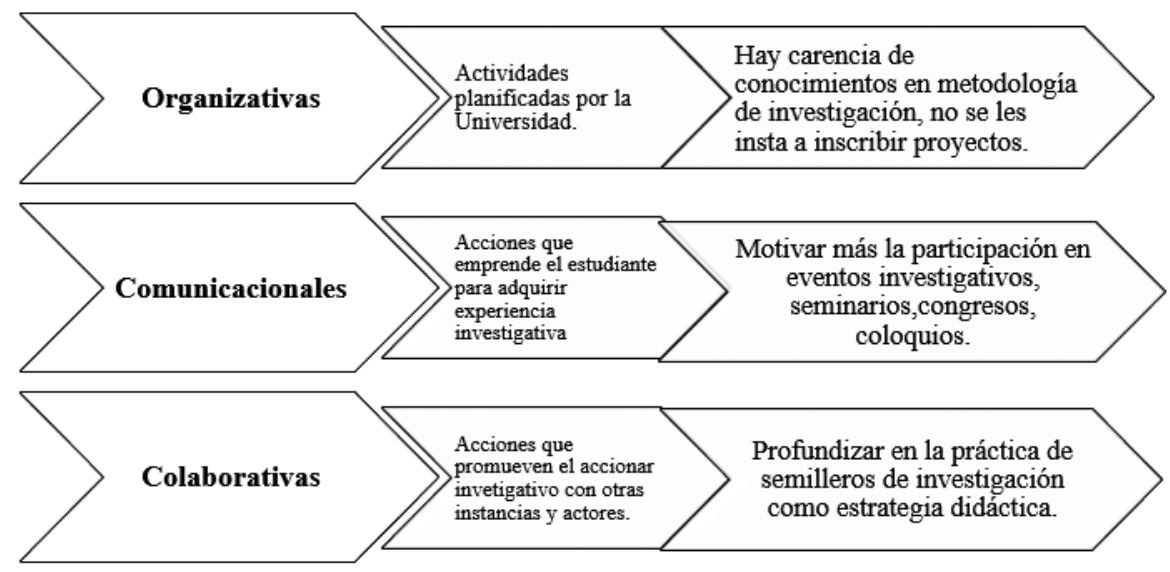

Figura 2. Clasificación de las competencias.

Fuente: (Ávalos, 2017. Comunicación III Jornadas de Investigación UNED).

Como se aprecia desde la clasificación de las competencias, existen carencias en la formación metodológica, participación del estudiantado en espacios de investigación y no se le anima al desarrollo de investigación en colaboración con expertos; dichos aspectos se desagregan con precisión en la tabla 2, dejando claras las competencias en investigación que actualmente se encuentran debilitadas entre los estudiantes de la UNED y que requieren reforzarse. 
El desarrollo de competencias investigativas en la formación de estudiantes de la UNED de Costa Rica mediante la metodología Lean Startup

Carolina Ávalos Dávila y María luisa Sevillano García

Tabla 2. Competencias investigativas por reforzar.

\begin{tabular}{clcc}
\hline Competencias & \multicolumn{1}{c}{ Indicadores } & Si & No \\
\hline \multirow{2}{*}{ Organizativas } & Metodología de Investigación & 63 & 138 \\
& Elaboración de proyectos institucionales & 67 & 134 \\
\hline \multirow{2}{*}{ Comunicacio- } & Participación en eventos de investigación & 63 & 139 \\
nales & Participación en investigación y extensión & 64 & 137 \\
& Publicación de investigaciones & 64 & 137 \\
\hline \multirow{2}{*}{ Colaborativas } & Semilleros de investigación & 62 & 139 \\
& Participación en eventos académicos como oyente & 50 & 151 \\
& Socialización del conocimiento investigativo & 67 & 134 \\
\hline
\end{tabular}

Fuente: (Ávalos, 2017. Comunicación III Jornadas de Investigación UNED).

Los hallazgos encontrados en la investigación, resaltaron la necesidad de robustecer en la adquisición de conocimientos en metodología de investigación y que desde la universidad se genere una cultura de incorporación del estudiantado en la participación e inscripción de proyectos de investigación.

Por otro lado, es necesario que se dé la participación de los estudiantes en eventos investigativos como: congresos, seminarios o coloquios, que le aporten mayor conocimiento en la temática de investigación; de igual forma, instar a los estudiantes a participar en proyectos de extensión, realizados en colaboración con su comunidad de aprendizaje.

Así mismo instar, al desarrollo de las prácticas investigativas proactivas, tal es el caso de los semilleros de investigación - (estrategia pedagógica usada para la formación de competencias en investigación, iniciada en Colombia en el año 1997) - , de forma que se involucre a los estudiantes en procesos de investigación desde un temprano proceso de formación, donde pasen de ser oyentes y socializadores a productores de propuestas de interés científico e innovador que vayan dando más cuerpo a su formación profesional. (Maldonado et al., 2007).

Ante un panorama en donde más del $50 \%$ de la población consultada indica que resaltan los problemas en la formación de investigación al finalizar el estudio de las asignaturas, es clara la necesidad de desarrollar acciones en función de nuevas metodologías de aprendizaje que promuevan acciones en pro de los vacíos encontrados.

Por ello, dejando claro que el desarrollo de una metodología de aprendizaje sustentada en los planteamientos del método Lean Startup, va a promover la formación de habilidades y destrezas emprendedoras, que además son consideradas como "competencias blandas" suscepti- 
bles de ser desarrolladas en el presente siglo, según J. Fernández (2012). Entonces, resulta interesante destacar cuáles son esas competencias evidenciadas y los beneficios que ofrecerían a los estudiantes de la UNED en el logro de una formación investigadora.

Posada (2005), establece, que el desarrollo de las competencias en el proceso de aprendizaje demanda por parte del alumnado, una gran voluntad de saber, así mismo señala, la importancia de que se mantenga una disciplina y voluntad de trabajo permanente, esforzándose por incrementar con espíritu crítico y constructivo, para integrar fuera del aula ese conocimiento adquirido.

Lo anterior sugiere, que el proceso de aprendizaje sea vivido con compromiso y constancia, para desarrollar acciones que propicien cambios de actitud en el desarrollo de competencias por parte del estudiantado, y hasta en los docentes, promoviendo la comprensión, pensamiento crítico, investigativo y creativo (Sevillano, 2015); en función de un conocimiento promotor del saber conocer, pensar, ser, convivir, sentir y compartir. Delors (1966), (citado en Posada, 2005), como se puede ver representado en la figura 3.

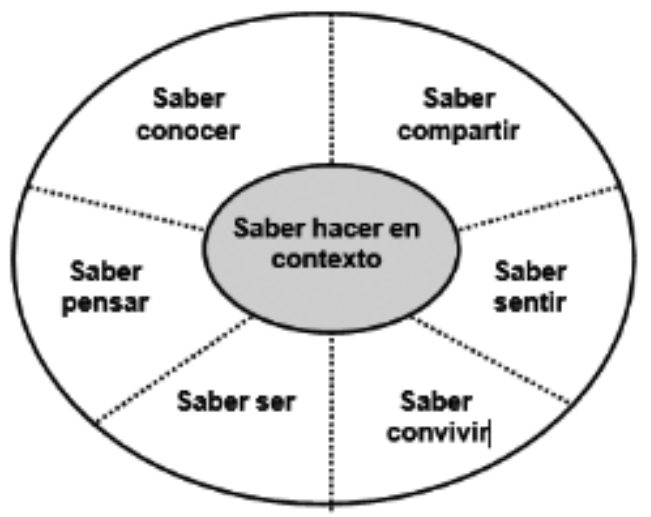

Figura 3. Sentido de las competencias. Posada (2005).

Para Alfaro (2015), las competencias emprendedoras, ... "son aqueIlas cualidades que debe tener una persona para iniciar y liderar cambios en su entorno, tanto en el plano personal como profesional y empresarial" ... (p.6).

Por otro lado, una actitud emprendedora resalta a aquella persona "... con capacidades de transformar, generar bienes y servicios de forma 
creativa, metódica, ética, responsable y efectiva" (Aguirre, 2017, párr.3).

De esa forma, solo un profesional con habilidades y destrezas conducentes al emprendimiento, que crea que la educación es para todos, podrá suscitar cambios significativos en el aprendizaje, ver en los diferentes momentos de socialización del conocimiento, una oportunidad para la investigación, reflexión y el aporte de ideas innovadoras. Aplicar los saberes teóricos logrados en la resolución de situaciones reales, de buscar las oportunidades para lograr un aprendizaje colaborativo, perdurable y de significado, a lo largo de su formación universitaria.

Estudios realizados por diversos autores expertos en el tema de emprendimiento, como: Ghina, Simatupang y Gustomo (2014) de Indonesia; Stayton \& Mangematin (2016) de Estados Unidos y Gámez, Saiz y Gil (2017) de Colombia, coindicen en que la comunicación, trabajo en equipo, proactividad, creatividad, resolución de problemas y el aprender a aprender, figuran como las principales competencias emprendedoras que todo talento humano debe desarrollar, datos que se ven reflejados en la siguiente tabla:

Tabla 3. Competencias emprendedoras.

\begin{tabular}{|c|c|}
\hline $\begin{array}{l}\text { Competencia } \\
\text { emprendedora }\end{array}$ & ¿Cómo se desarrolla? \\
\hline Comunicación & 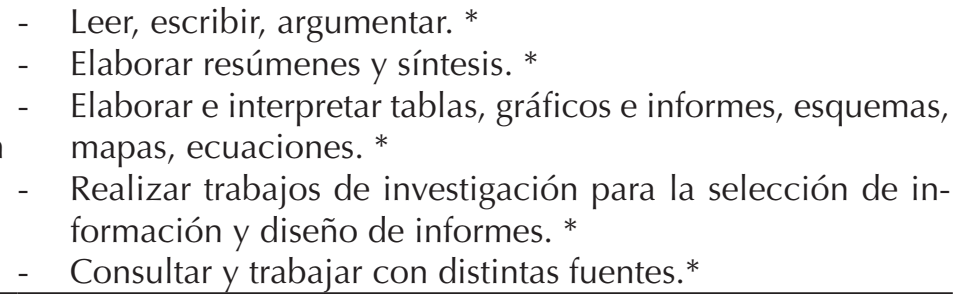 \\
\hline $\begin{array}{l}\text { Trabajo en } \\
\text { equipo }\end{array}$ & 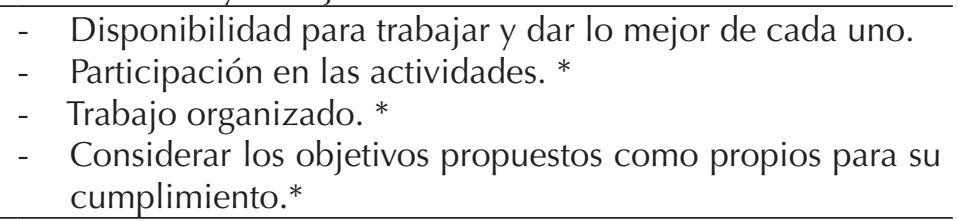 \\
\hline Proactividad & $\begin{array}{l}\text { - Toma de iniciativa y se emprenden las lecciones. * } \\
\text { - Perseverancia en las actividades por desarrollar. } \\
\text { - } \quad \text { Búsqueda constante de información sobre el área de traba- } \\
\text { jo que nos vincula. } \\
\text { - Interpretación y explicación efectiva de la información so- } \\
\text { licitada. * } \\
\text { - Afrontar positivamente el cambio.* }\end{array}$ \\
\hline
\end{tabular}




\begin{tabular}{|c|c|}
\hline Creatividad & $\begin{array}{ll}\text { - } & \text { Actualización en temas de la especialidad. }{ }^{*} \\
\text { - } & \text { Ampliación de los conocimientos y experiencia. }{ }^{*} \\
\text { - } & \text { Resolución de problemas de la especialidad. }{ }^{*} \\
& \text { Investigar y adoptar nuevas prácticas o métodos para resol- } \\
\text { - } & \text { Atener las necespecíficos. }{ }^{*} \\
& \text { tando soluciones a sus problemas. } \\
\text { - } & \text { Aplicación del conocimiento adquirido en diferentes situa- } \\
& \text { ciones. } \\
\text { - } & \text { Saber pedir ayuda a otros para brindar soluciones.* }\end{array}$ \\
\hline $\begin{array}{l}\text { Resolución de } \\
\text { problemas }\end{array}$ & 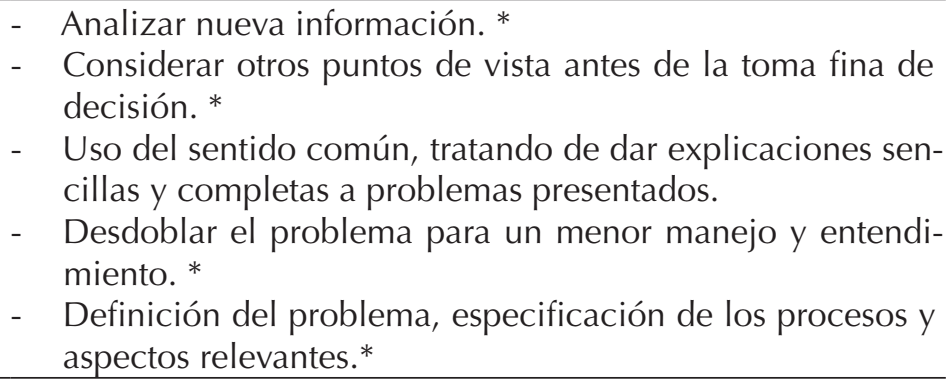 \\
\hline $\begin{array}{c}\text { Aprender a } \\
\text { aprender }\end{array}$ & 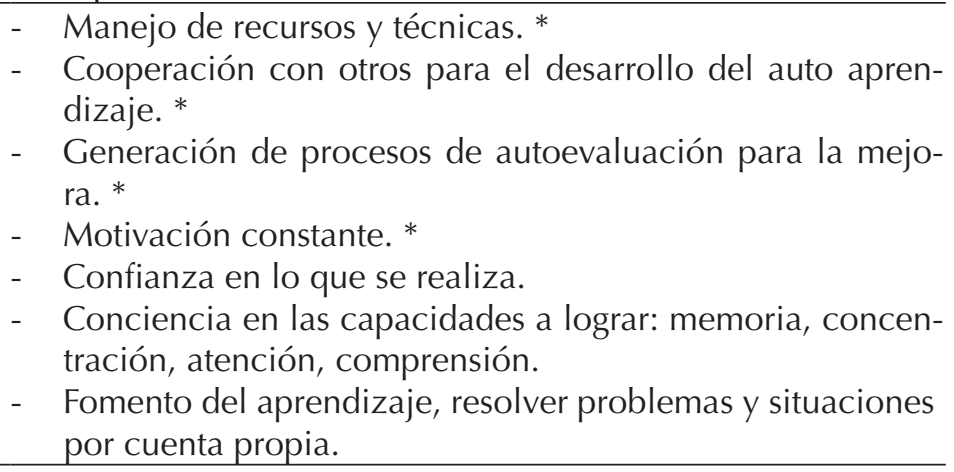 \\
\hline
\end{tabular}

(*): acciones investigativas presentes en cada competencia.

Fuente: Alfaro (2015), emprendedores juveniles de Nicaragua.

A partir de la información que se brinda en la tabla 3, es posible identificar en cada competencia emprendedora, la presencia de varias acciones investigativas las cuales se han resaltado con un $(*)$, esto con la intención de comprobar que en efecto, si se desarrolla una formación que involucre la adquisición de competencias emprendedoras, será posible lograr desde la formación del estudiantado el desarrollo de actitudes investigativas, pues es claro, que son requeridas para el cumplimiento de dichas competencias, y que una vez sistematizadas e interiorizadas, se convertirán en actitudes emprendedoras. 
Lo anterior hace suponer que, para lograr la formación en investigación a partir de la generación de actitudes emprendedoras ya interiorizadas por cada estudiante, es necesaria una labor muy proactiva por parte del docente y sobre todo del estudiante en el proceso formación. Un trabajo en colectivo, de autorregulación constante, de socialización, que se direccione a la aplicación del conocimiento logrado, a la búsqueda de información permanente, toma de decisiones, actitud muy participativa, capacidad de desarrollo de un pensamiento sintético, innovador y de gestión. Una flexibilidad para desarrollar diferentes procesos que se le soliciten y un respeto por las diferencias, resaltando valores como: tolerancia, respeto, ética, juicio, empatía, entre otros.

Dichos elementos en su conjunto, se ven representados en la figura 4, desde la cual se pretende, pasar de actitudes emprendedoras a formar habilidades investigativas en el estudiante de la UNED.

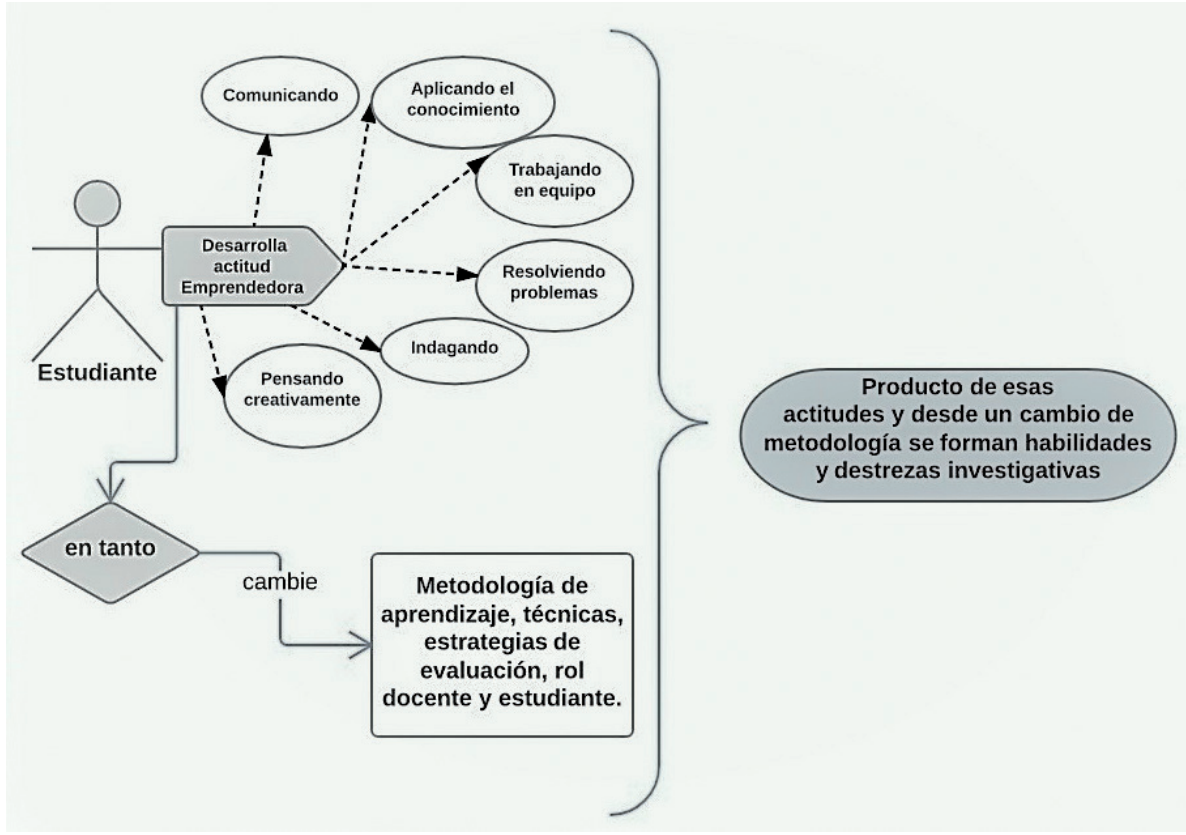

Figura 4. Actitudes emprendedoras y sus implicaciones para la formación en investigación.

Fuente: Elaboración propia.

Lograr una formación en investigación a partir de la generación de actitudes emprendedoras implica para el personal académico de la UNED 
de Costa Rica, un cambio notable en la metodología de aprendizaje, mayor compromiso desde la mediación por parte del profesor tutor, técnicas didácticas orientadas a la experimentación, trabajo colaborativo y mejoras en la evaluación empleada.

Por otro lado, es necesario contar con un refuerzo mayor por parte de la institución educativa en la promoción de actividades y espacios que inviten al estudiante a formar parte de procesos de investigación desde el inicio de su formación, donde tenga la oportunidad de realizar trabajo de campo, conozca y profundice en metodología investigativa, socialice hallazgos, producto de un esfuerzo colectivo que sea de mejora y beneficio para la carrera de la cual proviene.

Todos estos esfuerzos, favorecerán una actitud más participativa por parte del estudiante y el desarrollo de un sentido de pertenencia a la universidad, donde podrán sensibilizarse en razón de las necesidades reales de cada comunidad y buscar así, soluciones de mejora mediante una práctica investigativa razonada desde las bases de una mente emprendedora.

\section{Desarrollo de Competencias Investigativas a partir de Lean Startup como Metodología de aprendizaje}

Para el logro de dicho planteamiento y en razón de los aportes expuestos por Peñalver (2017), sobre la forma de llevar al aula el método Lean Startup como metodología de aprendizaje, debe existir una relación estrecha entre el método didáctico, las técnicas didácticas y heurísticas, etapas del método de base (en este caso el Lean Startup), la visión desde cómo se llevará a cabo la mediación en el proceso de aprendizaje y claramente, las competencias a mejorar, durante el proceso de formación.

El conglomerado de elementos educativos anteriores, son los que darán cuerpo a una metodología de aprendizaje sustentada en los planteamientos del método Lean Startup, aspectos representados en la figura 5: 


\section{Lean Startup en Educación}

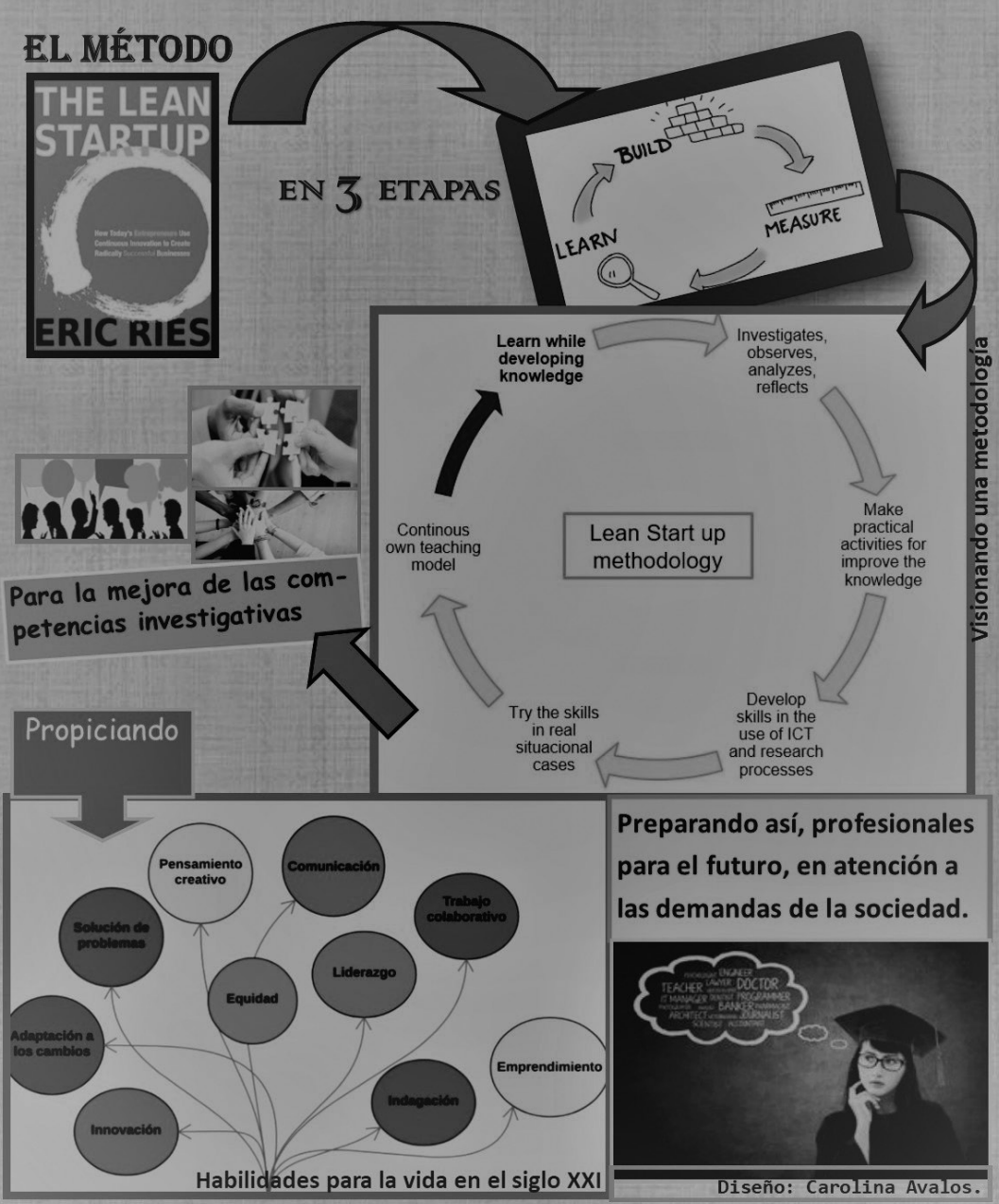

Figura 5: Desarrollo del método Lean Startup en Educación. Fuente: Elaboración propia.

La filosofía que plantea el método Lean Startup en sus tres etapas y concebida como metodología de aprendizaje en las aulas, puede verse materializada en los procesos de aprendizaje de la siguiente forma: 
CREAR (Build): en la medida en que las actividades y metodología desarrolladas en las asignaturas se orienten a generar espacios de aprendizaje donde se vayan robusteciendo las competencias investigativas identificadas: organizativas, comunicacionales y colaborativas, se va construyendo el conocimiento a partir técnicas didácticas que refuercen capacidades de observación, indagación, análisis y reflexión. El estudiante será capaz de aplicar tales capacidades en actividades prácticas que le reten a desarrollar casos aplicados y situacionales de acuerdo a su contexto, adicionalmente en el trabajo metodológico donde el docente sea impulsor y facilitador de oportunidades para que el estudiante desarrolle durante su proceso de formación habilidades tecnológicas en procesos investigativos; herramientas que le permitan profundizar tanto en teoría como en la comprensión metodológica. (Seas, 2016).

Por su parte, el docente, como agente facilitador, posibilitará el desarrollo de otras habilidades y destrezas tales como pensamiento crítico, trabajo de grupo, liderazgo, originalidad, creatividad y valores que transversalizan la enseñanza, como el respeto, tolerancia, responsabilidad, ética investigativa, entre otros.

MEDIR (Measure): Desde la puesta en marcha de las actividades y metodología llevada a cabo, así como el seguimiento de los aprendizajes logrados. Tanto el docente como los estudiantes, reconocen las fortalezas y debilidades logradas en el proceso de formación y las identifican, desde actividades de autoevaluación, coevaluación y evaluación unidireccional, desde el uso de diferentes técnicas de recolección de evidencias a lo largo del proceso de formación, tales como: escalas de observación y matrices tipo FODA, para informar al docente de los aspectos para mejorar en los siguientes periodos. (Rojas, 2016).

APRENDER (Learn): Siendo este un proceso logrado en conjunto por el estudiante y el docente, en donde cada uno desde su individualidad asume comprender la esencia del contenido visto y ser capaz de aplicar la teoría y buenas prácticas logradas a otros espacios individuales o colectivos. Convirtiéndose en agentes de cambio y de innovación en diferentes escenarios de formación, robusteciendo con ello, su modelo didáctico propio y carrera profesional.

En palabras de Gimeno (2008) citado por M. Fernández y Alcaraz (2016), un aprendizaje que resulte innovador y moderno, ..." donde no se entienda por moderno solo lo reciente o novedoso, si no lo que perdure para transformar la vida y la realidad..." (p.27). Es lo que se requie- 
re para la educación del futuro, alcanzando con ello un aprendizaje perdurable, transformable, aplicable y de provecho en diversas áreas, pero a la vez contribuyentes al campo de la docencia.

Considerando los planteamientos anteriores, y con el propósito de esclarecer cómo podría ser visualizada la nueva metodología de aprendizaje para el logro de una formación investigadora, se presenta la siguiente ilustración (ver figura 6), con una metodología de implementación, técnicas didácticas a emplearse desde la mediación, modelo de evaluación a considerar, así como las implicaciones de considerar una nueva metodología de aprendizaje sustentada en la filosofía del método Lean Startup. 
El desarrollo de competencias investigativas en la formación de estudiantes de la UNED de Costa Rica mediante la metodología Lean Startup Carolina Ávalos Dávila y María luisa Sevillano García

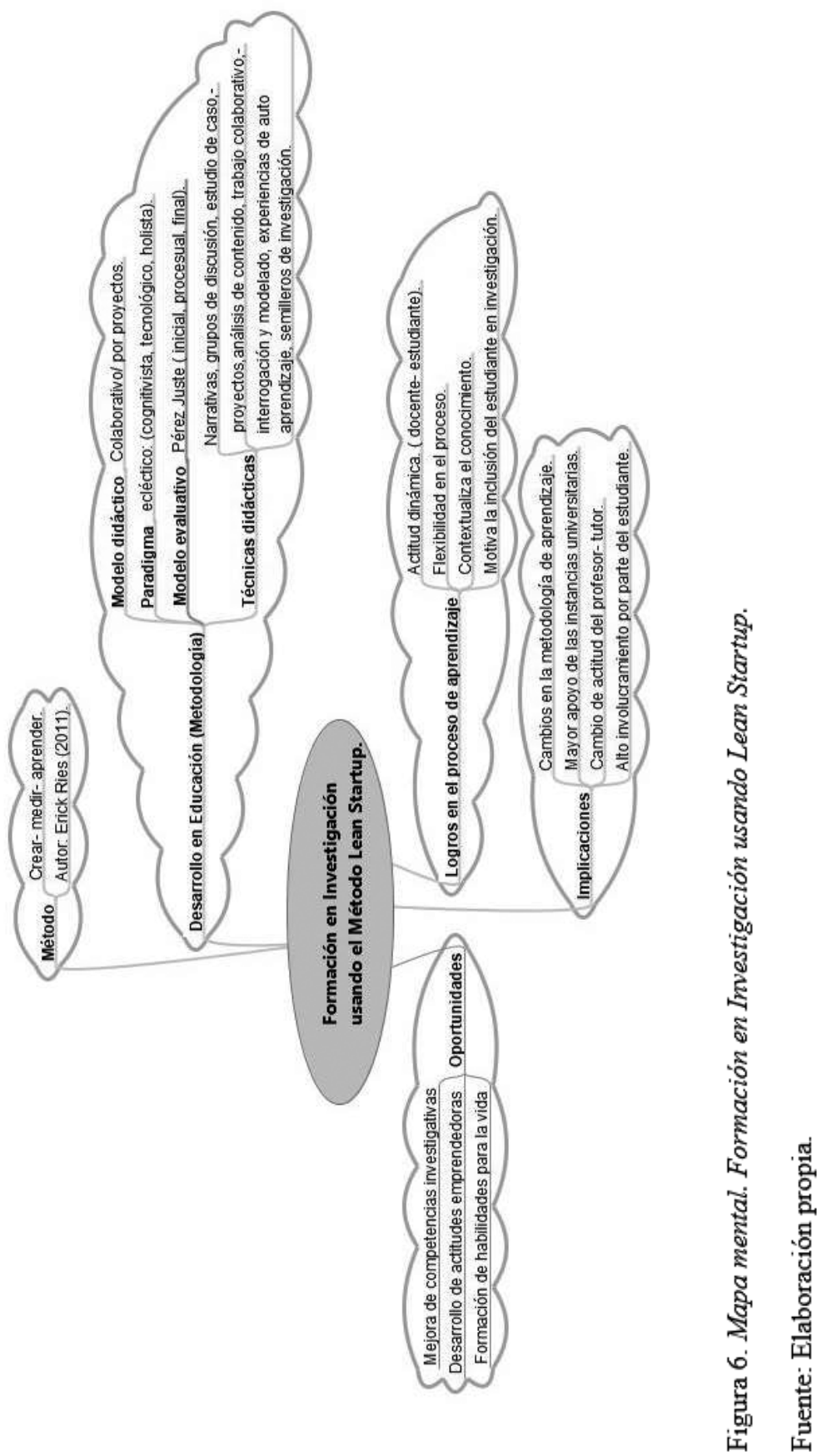




\section{Conclusiones}

Las aportaciones presentadas, en concordancia con los planteamientos de autores expertos en el tema de Lean Startup y emprendimiento, dejan entrever, que es posible lograr en este siglo, cambios significativos en la forma de enseñar y aprender si cada uno de los agentes educativos se predispone a una actitud innovadora, creativa y flexible; características claves de una "mente emprendedora", la cual debe estar presente en los procesos de formación de los futuros profesionales docentes de la UNED.

Lograr una formación en investigación como cualidad innata en los estudiantes universitarios desde el inicio de su carrera universitaria, implica, cambios importantes en las políticas educativas de educación superior, una mentalidad flexible desde las facultades universitarias, modificaciones en los diseños curriculares de planes, programas y asignaturas de estudio, donde se gestan los perfiles de salida de las diferentes carreras universitarias.

Se debe repensar el rol del profesor quién tutoriza las asignaturas, pero también, la función que tiene el estudiante y el compromiso que asume con su formación profesional. Consideramos importante que se le empiece a involucrar en temas de investigación desde el inicio de su formación, no debe dejarse esta práctica y experiencias únicamente a merced de los docentes e investigadores, puesto que la razón social y educativa de la universidad, desde los planteamientos de C. Tünnermann (2014), es garantizar la formación integral de profesionales que respondan y enfrenten las necesidades urgentes de la sociedad en la que se desarrollan. Y en estos momentos, la formación de competencias investigativas, así como las de emprendimiento, resultan ser claves para el desarrollo asertivo de los profesionales en el futuro.

Creemos en la importancia del entorno de aprendizaje como principal ecosistema de investigación, donde las posibilidades de generar propuestas de innovación se vean sistematizadas con el apoyo de todos los agentes sociales y educativos involucrados en procesos de formación profesional y académica.

Desde las evaluaciones, es necesario robustecer el seguimiento y realimentación que recibe el estudiante en su proceso de formación, pero para ello, deben ser importantes los retos educativos que demanden actividades prácticas donde se manifieste la puesta en marcha de soluciones 
reales mediante el estudio del contenido y la aplicación de ese conocimiento factual o memorístico. Han de ser actividades que sugieran un reto de estrategia, lógica y razonamiento.

Las conclusiones anteriores, urgen para un cambio en la gestión de los profesionales en educación, donde sea evidente una predisposición hacia las innovaciones, una responsabilidad frente a su formación permanente y claro está, una mente con proyección y creatividad donde se motiven a lograr una formación inclusiva, innovadora y de impacto para la generación de habilidades y destrezas investigativas, dando como resultado a un profesional proactivo, emprendedor, involucrado, con vocación, valores y generador de cambios a mediano y largo plazo.

Finalmente, para materializar las ideas anteriores, es necesaria la ruptura de paradigmas internos. Difícilmente se podrán generar procesos de innovación en el quehacer de cada profesional, si no se amplía el campo de acción, revisar con ímpetu prácticas novedosas y planteamientos que estén dando frutos importantes en otras disciplinas. Volcar la mirada a la forma en cómo se enseña y aprenden estudiantes de otras áreas de formación, con técnicas que hagan que el estudiante sea agente de cambio, con métodos que resulten ser adecuables a otros campos de formación y donde sus efectos sean efectivos o mejores. Tal es el caso del método Lean Startup, que nació en el área de Administración de Empresas, sin embargo, desde investigaciones y vivencias de docentes y estudiantes en Estados Unidos, Sudamérica, Europa y Asia, se ha visto su implementación y excelentes resultados en áreas como la docencia, ingeniería, agronomía, bibliotecología, entre otras.

\section{Referencias}

Ávalos, C. (mayo, 2017) Uso del método Lean Startup en el análisis y rediseño de estrategias didácticas para la formación en investigación. Comunicación, III Jornadas de Investigación. Doctorado en Educación. UNED. Madrid, España.

Aguirre, A. (18 de diciembre del 2017). Cultura de emprendimiento [Mensaje en un blog]@Gerencie.com. Recuperado de: https://www.gerencie.com/cultura-del-emprendimiento.html

Albert, G. M. J. (2009). La investigación Educativa. Claves Teóricas. España: Mc Graw-Hill.

Alfaro, C. (2015) ¿Cómo desarrollar competencias emprendedoras? Tips para adolescentes y jóvenes que quieren materializar sus ideas. UNICEF- Nicaragua. Imprime S.A. Recuperado de: http://unicef.org.ni/media/publicaciones/archivos/Caja_de_herramientas_para_emprendedores_adolescentes_y_j\%C3\%B3venes.pdf 
El desarrollo de competencias investigativas en la formación de estudiantes de la UNED de Costa Rica mediante la metodología Lean Startup

Carolina Ávalos Dávila y María luisa Sevillano García

Blank, S. (2013) Why the Lean Start- Up Changes Everything. Harvard Business. Retrieved from: https://hbr.org/2013/05/why-the-lean-start-up-changes-everything

Borafull, I. (2014). El futuro de la educación vinculado a un nuevo modelo productivo en una sociedad de cambios disruptivos. Revista de Humanidades; 13(2),150165. Recuperado de: https://www.fundacionpfizer.org/sites/default/files/pdf/dendra nov_2014_01_El_futuro_de_la_educacion.pdf

Calderón, K. (2013) Plan de desarrollo académico Universidad Estatal a Distancia. UNED 2012-2017. Recuperado de:

http://www.uned.ac.cr/academica/plan_academico/insumos/PlanDesAcad_UNED_ final.pdf

Colapinto, R. (2013) A combo of common sense and cutting edge, INNOVATION ACCOUNTING is the latest strategy to test a startup's odds for success. CAmagazine. Retrieved from: http://www.magazine-awards.com/index.php?tmpl=util_pdf_force_ inline\&file=http://www.magazine-awards.com/multimedia/krwawards_assets/assets/ files/awards/5/2992.pdf

Consejo Nacional de Rectores. (2016). Oficina de Planificación de la Educación Superior Costa Rica. Plan Nacional de la Educación Superior Universitaria Estatal 2016-2020. Recuperado de: http://siesue.conare.ac.cr/images/documentos/planes_2016_2020. pdf

Cooper, B. y Vlaskovits, P. (2014). El emprendedor Lean. Cómo los visionarios crean productos, desarrollan proyectos innovadores y transforman los mercados. Primera Edición. España. Universidad Internacional de La Rioja, S.A.

Cuevas, L., Guillén, D. y Rocha, V. (2011). Las competencias en investigación como puentes cognitivos para un aprendizaje significativo. Razón y Palabra. Recuperado de: http://www.redalyc.org/articulo.oa?id=199520010084

Davidson, Z. \& Palermo, C. (2015) Developing Research Competence in Undergraduate Students through Hands on Learning. Journal of Biomedical Education, 1,1-9. DOI:10.1155/2015/306380

Fernández, J. (2012). Emprendimiento y desarrollo de competencias blandas por alumnos de Ingeniería Comercial. Universidad de La Serena. Revista universitaria Ruta. 14, 41-56. Recuperado de: http://revistas.userena.cl/index.php/ruta/article/view/190

Fernández, M., Alcaraz, N. (2016) Innovación educativa. Más allá de la ficción. Madrid, España. Pirámide.

Gámez, J., Saiz, J. \& Gil, G. (2017) A cognitive, Emotional and Behavioral Assessment of Colombian Entrepreneurs Attitudes Toward Corruption. Universidad \& Empresa, 19, 1-33. Retrieved from: https://revistas.urosario.edu.co/xml/1872/187251252002/ index.html

Ghina, A., Simatupang, T. M., \& Gustomo, A. (2014). A Systematic Framework for Entrepreneurship Education within a University Context. International Education Studies, 7(12), 1-19. Retrieved from: https://eric.ed.gov/?id=EJ1071189

GÓMEZ, M., GALEANO, C. Y JARAMILLO, D (2015). El estado del arte: una metodología de investigación. Revista Colombiana de Ciencias Sociales, 6(2), 423-442.

GUERRERO, M. (2007). Formación de habilidades para la investigación desde el pregrado. Acta Colombiana de Psicología, 10(2), 190-192. Retrieved from: http://www. 
El desarrollo de competencias investigativas en la formación de estudiantes de la UNED de Costa Rica mediante la metodología Lean Startup Carolina Ávalos dávila y María luisa Sevillano García

scielo.org.co/scielo.php?script=sci_arttext\&pid=S012391552007000200018lng=en \&tlng $=\mathrm{es}$.

Gupte, A. (2015). The Very Lean Startup Method. Journal for Contemporary Research in Management. 7, 52-57. Retrieved from: http://dawnsvims.in/wp-content/ uploads/2015/11/7-The-Very-Lean-Startup-Method-Aniruddha-Gupte.pdf

Hetch, Y. (2010) Democratic education, a Beginning of a Story, Israel: AERO.

Maldonado, L; Landazábal, D; Hernández, J, et al. (2007). Visibilidad y formación en Investigación. Estrategias para el desarrollo de competencias investigativas. Universidad Nacional Abierta y a Distancia UNAD (Bogotá - Colombia). Revista Studiositas. 2(2), $43-56$.

Moreno, G. (2005). Potenciar la educación. Un curriculum transversal de formación para la investigación. REICE. Revista electrónica Iberoamericana sobre Calidad, Eficacia y Cambio en Educación; 3(1), 1-22. Recuperado de: http://www.redalyc.org/articulo. oa? id=55130152

Peñalver, P. (2017). Lean Startup en Educación. Emprender no es una opción. PDF.

Posada, R. (2005). Formación Superior basada en competencias, interdisciplinariedad y trabajo autónomo del estudiante. Revista Iberoamericada de Educación. 34, 1-34. Recuperado de: http://www.rieoei.org/deloslectores/648Posada.PDF

Ries, E. (2011). The Lean Startup. How today's entrepreneurs use continuous innovation to create radically successful businesses. $1^{\text {st }}$ Ed. New York. Crown Business.

Rojas, A. (2016). Planeamiento del Proceso de Enseñanza-Aprendizaje. San José, Costa Rica. EUNED.

Seas, J. (2016). Didáctica General I. San José, Costa Rica. EUNED.

Sevillano, M. (2011). Didáctica en el núcleo de la Pedagogía. Tendencias Pedagógicas, 18, 7-32. Recuperado de: https://dialnet.unirioja.es/descarga/articulo/3778534.pdf

Sevillano, M. y Vázquez E. (2015). Modelos de investigación en contextos móviles y ubicuos en Educación Superior. Madrid. McGraw-Hill.

Shank, R (2011) Teaching Minds: How Cognitive Science Can Save Our Schools. New York Teachers College Press.

Sousa, P.; Cavalcanti, J. (2016), Analysis of Lean Innovation Management Model. Revista Gestão.Org, 14, 277-287. Retrieved from: http://www.revista.ufpe.br/gestaoorg

Stayton, J. \& Mangematin, V. (2016) Startup time, innovation and organizacional emergence: A study of USA-based international technology ventures. Journal of International Entrepreneurship; 14, 373. DOI: https://doi.org/10.1007/s10843-016-0183-y

Tobón, S. (2013). Formación integral y competencias. Pensamiento complejo, currículo, didáctica y evaluación (4ta. Ed.) Bogotá. ECOE. Recuperado de: https:// www.researchgate.net/profile/Sergio_Tobon4/publication/319310793_Formacion_ integral_y_competencias_Pensamiento_complejo_curriculo_didactica_y_evaluacion/links/59a2edd9a6fdcc1a315f565d/Formacion-integral-y-competencias-Pensamiento-complejo-curriculo-didactica-y-evaluacion.pdf

Tünnermann, C.; Souza, M. (2003). Desafíos de la Universidad en la Sociedad del Conocimiento, Cinco Años Después de la Conferencia Mundial sobre Educación Superior. UNESCO Forum on Higher Education, Research and Knowledge. Recuperado de: http://unesdoc.unesco.org/images/0013/001344/134422so.pdf 
El desarrollo de competencias investigativas en la formación de estudiantes de la UNED de Costa Rica mediante la metodología Lean Startup

Carolina Ávalos Dávila y María luisa Sevillano García

Tünnermann, C. (2014) Mi visión de la universidad del futuro. Instituto para el Desarrollo de la Investigación Educativa en Iberoamérica S.C. e- Revista IDIIEI. Recuperado de: http://idiiei.org/index.php/articulos/item/mi-vision-de-la-universidad-del-futuro 